PROCEEDINGS OF THE

AMERICAN MATHEMATICAL SOCIETY

Volume 128, Number 10, Pages 2863-2868

S 0002-9939(00)05339-9

Article electronically published on March 29, 2000

\title{
ARE COVERING (ENVELOPING) MORPHISMS MINIMAL?
}

\author{
EDGAR E. ENOCHS, J. R. GARCÍA ROZAS, AND LUIS OYONARTE
}

(Communicated by Wolmer V. Vasconcelos)

\begin{abstract}
We prove that for certain classes of modules $\mathcal{F}$ such that direct sums of $\mathcal{F}$-covers $(\mathcal{F}$-envelopes $)$ are $\mathcal{F}$-covers $(\mathcal{F}$-envelopes $), \mathcal{F}$-covering $(\mathcal{F}$ enveloping) homomorphisms are always right (left) minimal. As a particular case we see that over noetherian rings, essential monomorphisms are left minimal. The same type of results are given when direct products of $\mathcal{F}$-covers are $\mathcal{F}$-covers. Finally we prove that over commutative noetherian rings, any direct product of flat covers of modules of finite length is a flat cover.
\end{abstract}

\section{Preliminaries}

This study was prompted by the following question. If $f: M \rightarrow M$ is an endomorphism of a (left) $R$-module such that the restriction of $f$ to some essential submodule $N$ is the identity on $N$, then is $f$ an automorphism of $M$ ?

In this paper we will show that the answer is yes if the ring $R$ is left noetherian. In fact we will note that this question is a special case of a more general one and that our techniques provide answers in the more general situation.

Definition 1.1 ([2, pgs. 6 and 8]). Let $R$ be any associative ring (not necessarily commutative). A morphism of $R$-modules $f: M_{1} \rightarrow M_{2}$ is said to be left (right) minimal if any endomorphism $g \in \operatorname{End}_{R}\left(M_{2}\right)$ (any $g \in \operatorname{End}_{R}\left(M_{1}\right)$ ) such that $g \circ f=f(f \circ g=f)$ is an automorphism.

Thus, the main concern of this paper will be the study of left (right) minimal homomorphisms.

All rings considered in this paper will be associative and, unless otherwise specified, not necessarily commutative. For any module $M, E(M)$ will denote the injective envelope of $M$, and if the $\operatorname{ring} R$ is local with maximal ideal $m, \hat{R}$ will denote the completion of $R$ with respect to the $m$-adic topology.

We will use the language of covers and envelopes from Xu [6]. (We note that these notions are also known as right and left approximations [1].)

The symbols $\mathcal{E}$ and $\mathcal{F}$ will denote classes of modules closed under isomorphisms, and such that $\mathcal{E}$ contains the class of injective modules and $\mathcal{F}$ contains the class of projective modules. Then it is well known that $\mathcal{E}$-envelopes are injective and $\mathcal{F}$-covers are surjective.

Received by the editors April 21, 1998 and, in revised form, November 14, 1998.

2000 Mathematics Subject Classification. Primary 16D10; Secondary 16D40, 13H99.

Key words and phrases. Essential submodule, superfluous submodule, cover, envelope, minimal homomorphism, covering homomorphism, enveloping homomorphism. 
The next definition, which was given in [5, will be very useful throughout this paper.

Definition 1.2. A morphism $f: M_{1} \rightarrow M_{2}$ is said to be $\mathcal{F}$-covering (or covering) if $M_{1}$ and $M_{2}$ have $\mathcal{F}$-covers $\varphi_{1}: F_{1} \rightarrow M_{1}$ and $\varphi_{2}: F_{2} \rightarrow M_{2}$ and if some (so every) lifting $g: F_{1} \rightarrow F_{2}$ is an isomorphism.

The morphism is said to be $\mathcal{E}$-enveloping if the dual situation holds.

Remark. Note that if $f: M_{1} \rightarrow M_{2}$ is any covering (enveloping) homomorphism and $\varphi_{1}: L_{1} \rightarrow M_{1}$ is any cover of $M_{1}\left(\varphi_{2}: M_{2} \rightarrow L_{2}\right.$ is any envelope of $\left.M_{2}\right)$, then $f \circ \varphi_{1}$ is a cover of $M_{2}\left(\varphi_{2} \circ f\right.$ is an envelope of $\left.M_{1}\right)$.

So our basic general question is whether an $\mathcal{F}$-covering ( $\mathcal{E}$-enveloping) morphism $f: M_{1} \rightarrow M_{2}$ is right (left) minimal.

\section{When $\mathcal{F}$-COVERs ( $\mathcal{E}$-envelopes) ARE Closed Under DiReCt SUMS}

In this section we will assume that the classes $\mathcal{E}$ and $\mathcal{F}$ are such that the direct sums of $\mathcal{E}$-envelopes and $\mathcal{F}$-covers are also $\mathcal{E}$-envelopes and $\mathcal{F}$-covers respectively. We will show that under these circumstances we can give a positive answer to our general question.

Definition 2.1. An endomorphism of modules $f: M \rightarrow M$ is said to be locally nilpotent on $M$, if for any $x \in M$ there exists a natural number $n$ such that $f^{n}(x)=0$.

Proposition 2.2. Let $M$ be any $R$-module and let $\varphi: M \rightarrow E$ be an $\mathcal{E}$-envelope. If $g \in \operatorname{End}_{R}(E)$ is such that $g \circ \varphi=\varphi$, then if $g=i d_{E}+\bar{g}, \bar{g}: E \rightarrow E$ is locally nilpotent on $E$.

Proof. It is clear that any $g \in \operatorname{End}_{R}(E)$ such that $g \circ \varphi=\varphi$ can be written as $g=i d_{E}+\bar{g}$ where $\bar{g} \in \operatorname{End}_{R}(E)$ and $\operatorname{Im}(\varphi) \subseteq \operatorname{Ker}(\bar{g})$.

Since direct sums of $\mathcal{E}$-envelopes are $\mathcal{E}$-envelopes, $\bigoplus_{\mathbb{N}} M \rightarrow \bigoplus_{\mathbb{N}} E$ is an $\mathcal{E}$ envelope. If we consider the map $h \in \operatorname{End}_{R}\left(\bigoplus_{\mathbb{N}} E\right)$, given by

$$
h\left(\left(x_{i}\right)_{i \in \mathbb{N}}\right)=\left(x_{0}, x_{1}-\bar{g}\left(x_{0}\right), x_{2}-\bar{g}\left(x_{1}\right), \ldots\right),
$$

we get $h \circ \bigoplus \varphi=\bigoplus \varphi$.

Thus the map $h$ is an automorphism and for any $x \in E$ there exists an element $\left(x_{i}\right)_{i \in \mathbb{N}}$ which is mapped onto $(x, 0,0, \ldots)$. It follows that $x_{n}=\bar{g}^{n}(x)$ for all $n$, so for $n$ sufficiently large $\bar{g}^{n}(x)=0$.

We note that the above result says that $E=\bigcup_{n=1}^{\infty} \operatorname{Ker}\left(\bar{g}^{n}\right)$, or equivalently that $E=\underline{\lim } \operatorname{Ker}\left(\bar{g}^{n}\right)$.

A proposition similar to the above also holds if we replace $\mathcal{E}$-envelopes by $\mathcal{F}$ covers. Then, if $\varphi: F \rightarrow M$ is an $\mathcal{F}$-cover and $g: F \rightarrow F$ is such that $\varphi \circ g=\varphi, g$ can be written as $g=i d_{F}+\bar{g}$ with $\bar{g}$ locally nilpotent on $F$.

Proposition 2.3. If $f: M_{1} \rightarrow M_{2}$ is an $\mathcal{E}$-enveloping homomorphism, then it is left minimal. 
Proof. Let $g \in \operatorname{End}_{R}\left(M_{2}\right)$ be such that $g \circ f=f$. Then, if $\varphi: M_{2} \rightarrow E$ is an $\mathcal{E}$-envelope, the diagram

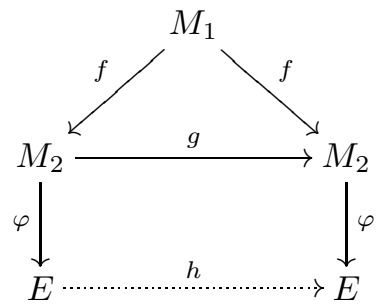

can be completed commutatively by a morphism $h$. This $h$ is an automorphism because $f$ is enveloping, so the map $\varphi \circ f$ is an envelope.

Now, since $\varphi \circ f$ is an envelope, by Proposition 2.2 we get that $h=i d_{E}+\bar{h}$ with $\bar{h}$ locally nilpotent on $E$. Let $g=i d_{M_{2}}+\bar{g}$. The equality $\varphi \circ g=h \circ \varphi$ gives the equality $\varphi \circ \bar{g}=\bar{h} \circ \varphi$.

Therefore, for any $x \in M_{2}$ and any $n \in \mathbb{N}$ we have $\left(\varphi \circ \bar{g}^{n}\right)(x)=\left(\bar{h}^{n} \circ \varphi\right)(x)$, so, for $n$ sufficiently large we get $\left(\varphi \circ \bar{g}^{n}\right)(x)=0$ because $\bar{h}$ is locally nilpotent on $E$. Thus we get that $\bar{g}^{n}(x)=0$ since $\varphi$ is injective, and so $\bar{g}$ is locally nilpotent on $M_{2}$.

The fact that $\bar{g}$ is locally nilpotent on $M_{2}$ says that the map

$$
i d_{M_{2}}+\sum_{i \geq 1}(-1)^{i} \bar{g}^{i}: M_{2} \rightarrow M_{2}
$$

is well defined. It is straightforward to prove that this morphism is the inverse of $g$.

As an application we return to the problem mentioned in the preliminary section. We are now able to say that over a left noetherian ring, the answer to this problem is always yes.

Corollary 2.4. Let $R$ be a left noetherian ring. If $M_{1}$ is an essential submodule of a left $R$-module $M_{2}$ and $f \in \operatorname{End}_{R}\left(M_{2}\right)$ is the identity on $M_{1}$, then $f$ is an automorphism.

Proof. If the ring is noetherian, then any direct sum of injective envelopes is an injective envelope. Moreover, the inclusion $M_{1} \hookrightarrow M_{2}$ is an enveloping morphism (cf. [5, pg. 6]), so then apply Proposition 2.3.

The same type of results holds for covering morphisms. Following the same type of arguments we get.

Proposition 2.5. If $f: M_{1} \rightarrow M_{2}$ is an $\mathcal{F}$-covering homomorphism, then $f$ is right minimal.

Corollary 2.6. Let $R$ be a left perfect ring. If $S \leq M$ is a superfluous submodule of a left $R$-module $M$ and $f \in \operatorname{End}_{R}(M)$ is such that $p \circ f=f$ (note that $p: M \rightarrow \frac{M}{S}$ is the canonical projection), then $f$ is an automorphism.

Proof. If $R$ is left perfect, every left $R$-module has a projective cover and any direct sum of projective covers is a projective cover. Moreover the projection $p$ is in this case a covering morphism (cf. [5, Proposition 3.2]), so apply Proposition 2.5.

It is an interesting question whether Corollaries 2.4 and 2.6 hold for more general rings other than left noetherian and left perfect respectively. The general question is whether $\mathcal{E}$-enveloping and $\mathcal{F}$-covering morphisms are always minimal. 


\section{When PRODUCTS OF $\mathcal{F}$-COVERS ARE $\mathcal{F}$-COVERS}

In this section $\mathcal{F}$ will denote a class of modules such that any direct product of $\mathcal{F}$-covers is an $\mathcal{F}$-cover.

It is easy to prove the next proposition by using dual arguments to these of Proposition 2.2, and the homomorphism

$$
\xi \in \operatorname{End}_{R}\left(\prod F\right) \xi\left(x_{0}, x_{1}, \ldots\right)=\left(x_{0}-\bar{f}\left(x_{1}\right), x_{1}-\bar{f}\left(x_{2}\right), \ldots\right)
$$

instead of the given $h \in \operatorname{End}_{R}\left(\bigoplus_{\mathbb{N}} F\right)$ in Proposition 2.2.

Proposition 3.1. Let $\varphi: F \rightarrow M$ be an $\mathcal{F}$-cover. Let $f \in \operatorname{End}_{R}(F)$ be such that $\varphi \circ f=\varphi$ and let $f=i d_{F}+\bar{f}$ (so that $\operatorname{Im}(\bar{f}) \subseteq \operatorname{Ker}(\varphi)$ ). If $\bigcap \operatorname{Im}\left(\bar{f}^{n}\right)=0$, then $F \cong \lim _{\operatorname{Coker}}\left(\bar{f}^{n}\right)$. If $\bar{f}$ is an injection, then $\bigcap \operatorname{Im}\left(\bar{f}^{n}\right)=0$.

Example. Let $R$ be an integral domain such that every nonzero torsion module has a simple submodule. Then any direct product of torsion free covers is a torsion free cover (4 Theorem 4.1]). Let $r \in R^{*}$ and let $M$ be an $R$-module such that $r M=0$.

If $\varphi: F \rightarrow M$ is a torsion free cover, let us define the morphism $f: F \rightarrow F$, $f(x)=x+r x$. It is clear that $\operatorname{Im}(\bar{f}) \subseteq \operatorname{Ker}(\varphi)$ and that $\bar{f}$ is an injection; then by Proposition $3.1 F \cong \varliminf_{\varlimsup} \frac{F}{r^{n} F}$.

Let us now prove the dual result of Proposition 2.5 for which we will need the following lemma.

Lemma 3.2. Let $f: M_{1} \rightarrow M_{2}$ be a covering homomorphism and let $g: M_{1} \rightarrow$ $M_{1}$ be such that $f \circ g=f$. If $g=i d_{M_{1}}+\bar{g}$ and if $\bigcap \operatorname{Im}\left(\bar{g}^{n}\right)=0$, then $M_{1} \cong$ $\varliminf$ Coker $\left(\bar{g}^{n}\right)$.

Proof. If we note that $\prod f: \prod_{n \in \mathbb{N}} M_{1} \rightarrow \prod_{n \in \mathbb{N}} M_{2}$ is also covering, we see that a straightforward modification of the proof of Proposition 3.1 gives the desired result.

Proposition 3.3. Let $f: M_{1} \rightarrow M_{2}$ be an $\mathcal{F}$-covering homomorphism such that for every homomorphism $g: M_{1} \rightarrow M_{1}$ with $f \circ g=f$ it is true that $\bigcap \operatorname{Im}\left(\bar{g}^{n}\right)=0$ when $g=i d_{M_{1}}+\bar{g}$. Then $f$ is right minimal.

Proof. By Lemma $3.2 M_{1} \cong \lim _{\operatorname{Coker}}\left(\bar{g}^{n}\right)$ so $M_{1}$ is complete with the topology having the $\operatorname{Im}\left(\bar{g}^{n}\right)$ as neighborhoods of 0 . Then if $t: M_{1} \rightarrow M_{1}$ is defined by $t=\sum_{n \geq 0}(-1)^{n} \bar{g}^{n}$ we see that $t$ is well defined. Then it is easy to check that $t=g^{-1}$ and so $g$ is an automorphism.

At this point it is natural to raise the question of which conditions (on the ring or on the class) guarantee that a direct product of $\mathcal{F}$-covers is still an $\mathcal{F}$-cover. This question has been studied for certain classes of modules over particular rings; for example, the class of torsion free modules over integral domains 4 . Using the techniques developed in this paper we give another partial result in this direction in Theorem 3.7. We will consider the class $\mathcal{F}$ of flat modules over a commutative, noetherian ring. We recall that a left $R$-module $C$ (over any $R$ ) is said to be cotorsion if $\operatorname{Ext}^{1}(F, C)=0$ for each flat left $R$-module $F$.

The proof of the following lemma is straightforward.

Lemma 3.4. Let $M$ be a module over a commutative ring $R$, and let $E$ be an injective R-module. Then the module $\operatorname{Hom}_{R}(M, E)$ is cotorsion. 
Theorem 3.5. Let $R$ be a local commutative noetherian ring and $A$ an $R$-module of finite length. Then there exists a morphism $\varphi: \hat{R}^{n} \rightarrow A$ which is a flat cover of $A$ as an $R$-module, for some $n \in \mathbb{N}$. Furthermore, $\operatorname{Ker}(\varphi) \subseteq \prod_{i=1}^{n} m \hat{R}$.

Proof. Since $A$ is of finite length, it is an $\hat{R}$-module and, furthermore, as an $\hat{R}$ module it is Matlis reflexive. We know that over a local ring every finitely generated module has a projective cover, which is actually a free cover since over local rings all projective modules are free. So there exists a projective cover $\varphi: \hat{R}^{n} \rightarrow A$ of $A$ as an $\hat{R}$-module.

Let $C$ be the kernel of such a cover. Then $C$ is a finitely generated $\hat{R}$-module, and so Matlis reflexive. Furthermore, $C^{\nu}$ is an artinian $\hat{R}$-module, which implies that $\operatorname{Hom}_{\hat{R}}\left(C^{\nu}, E\left(\frac{R}{m}\right)\right)=\operatorname{Hom}_{R}\left(C^{\nu}, E\left(\frac{R}{m}\right)\right.$ ) (note that the injective envelope of $\frac{R}{m}$ as an $R$-module is the same as an $\hat{R}$-module, so we can write $\left.E\left(\frac{R}{m}\right)\right)$, thus $C \cong C^{\nu \nu}=H_{o m}\left(C^{\nu}, E\left(\frac{R}{m}\right)\right)$. Therefore, by Lemma 3.4 $C$ is a cotorsion $R$ module, and then $\varphi: \hat{R}^{n} \rightarrow A$ is a flat precover of $A$ as an $R$-module.

Now it is known that any $R$-homomorphism $\hat{R}^{n} \rightarrow \hat{R}^{n}$ is also an $\hat{R}$-homomorphism, so since any $\hat{R}$-homomorphism $f: \hat{R}^{n} \rightarrow \hat{R}^{n}$ with $\varphi \circ f=\varphi$ is an automorphism, we get that $\varphi: \hat{R}^{n} \rightarrow A$ is in fact a flat cover of $A$ as an $R$-module.

For the rest note that $\varphi=\varphi_{1}+\cdots+\varphi_{n}$ where $\varphi_{i}=\varphi \circ k_{i}\left(k_{i}: \hat{R} \hookrightarrow \hat{R}^{n}\right.$ denotes the $i$ th injection). So $\operatorname{Ker}(\varphi) \nsubseteq \prod_{i=1}^{n} m \hat{R}$ if and only if $\varphi_{i}=0$ for some $i$. But in this case the morphism $f: \hat{R}^{n} \rightarrow \hat{R}^{n}$ given by $f\left(x_{1}, \ldots, x_{n}\right)=$ $\left(x_{1}, \ldots, x_{i-1}, 0, x_{i+1}, \ldots, x_{n}\right)$ is such that $\varphi \circ f=\varphi$, so that $f$ must be an automorphism, which it is not since it is not a monomorphism.

Corollary 3.6. Let $R$ be a commutative noetherian ring and $A$ an $R$-module of finite length. Then there exists a flat cover of $A$ of the form $\varphi: \bigoplus_{i=1}^{k} \hat{R}_{m_{i}}^{n_{i}} \rightarrow$ $A$ where $m_{i}$ are maximal ideals of $R$ and $k, n_{i} \in \mathbb{N}$. Furthermore $\operatorname{Ker}(\varphi) \subseteq$ $\bigoplus_{i=1}^{k}\left(\bigoplus_{j=1}^{n_{i}} m_{i} \hat{R}\right)$.

Proof. Since $A$ is of finite length, by the Chinese Remainder Theorem it can be written as $A=A_{1} \oplus \cdots \oplus A_{s}$, where each $A_{i}$ is annihilated by a power of some maximal ideal $m_{i}$ of $R$. Thus, to get a flat cover of $A$ we just need to find one for each $A_{i}$. But each $A_{i}$ is naturally an $R_{m_{i}}$-module. Hence $A_{i}$ as an $R_{m_{i}}$-module has a flat cover of the form $\hat{R}_{m_{i}}^{n_{i}} \rightarrow A_{i}$. But then it is easy to see that this is also a flat cover as an $R$-module. For if $F$ is a flat $R$-module, any linear $F \rightarrow A_{i}$ gives an $R_{m_{i}}$-linear $F_{m_{i}} \rightarrow\left(A_{i}\right)_{m_{i}}=A_{i}$. So, we can factor through $\hat{R}_{m_{i}}^{n_{i}} \rightarrow A_{i}$. Hence, we have a flat precover as $R$-modules. Since any $R$-linear $\hat{R}_{m_{i}}^{n_{i}} \rightarrow \hat{R}_{m_{i}}^{n_{i}}$ is also $R_{m_{i}}$-linear, in fact we have a flat cover as $R$-modules. If we now appeal to Theorem 3.5 and use the fact that finite sums of flat covers are flat covers (see 3 . Proposition 4.1] or [5, Proposition 2.8] for a simple proof) we get the result.

Theorem 3.7. Let $R$ be a commutative noetherian ring, and $\left\{A_{i} ; i \in I\right\}$ a family of $R$-modules of finite length. If $\varphi_{i}: F_{i} \rightarrow A_{i}$ is a flat cover for all $i$, then $\prod \varphi_{i}$ : $\prod_{i \in I} F_{i} \rightarrow \prod_{i \in I} A_{i}$ is a flat cover.

Proof. First we note that by the last corollary we can suppose $F_{i}=\bigoplus_{j=1}^{t_{i}} \hat{R}_{m_{i j}}^{\alpha_{i j}}$ and $\operatorname{Ker}\left(\varphi_{i}\right) \subseteq \bigoplus_{j=1}^{t_{i}} \bigoplus_{k=1}^{\alpha_{i j}} m_{i j} \hat{R}_{m_{i j}}$.

Now we know that the product of flat covers is a flat precover since the class of flat modules is closed under direct products ( $R$ is noetherian). We only need to 
show that if $f: \prod_{i \in I} \bigoplus_{j=1}^{t_{i}} \hat{R}_{m_{i j}}^{\alpha_{i j}} \rightarrow \prod_{i \in I} \bigoplus_{j=1}^{t_{i}} \hat{R}_{m_{i j}}^{\alpha_{i j}}$ is such that $\prod \varphi_{i} \circ f=\prod \varphi_{i}$, then $f$ is an automorphism.

Such a homomorphism $f$ can be written as $f=i d+\bar{f}$, where $\operatorname{Im}(\bar{f}) \subseteq \operatorname{Ker}\left(\prod \varphi_{i}\right)$ $\subseteq \prod_{i \in I} \bigoplus_{j=1}^{t_{i}} \bigoplus_{k=1}^{\alpha_{i j}} m_{i j} \hat{R}_{m_{i j}}$. Since $\hat{R}_{m_{i j}}$ is complete with respect to the $m_{i j}$-adic topology for all $i, j$, then $\prod_{i \in I} \bigoplus_{j=1}^{t_{i}} \hat{R}_{m_{i j}}^{\alpha_{i j}}$ is also complete. From this fact it is easy to see that $\prod_{i \in I} \bigoplus_{j=1}^{t_{i}} \hat{R}_{m_{i j}}^{\alpha_{i j}} \cong \varliminf_{\varlimsup} \operatorname{Coker}\left(\bar{f}^{n}\right)$.

Therefore, we can conclude that the map $g=\sum_{n \geq 0}(-1)^{n} \bar{f}^{n}$ is a well-defined morphism of $R$-modules, and $g=f^{-1}$, so $f$ is an automorphism.

\section{ACKNOWLEDGEMENTS}

The authors were partially supported by the NATO grant CRG 971543. The last two authors were also supported by the DGICYT grant PB91-1068.

\section{REFERENCES}

[1] M. Auslander and I. Reiten. Applications of Contravariantly Finite Subcategories. Cambridge Studies in Advanced Mathematics № 86, Cambridge University Press, Cambridge (1991), 111-152. MR 92e:16009

[2] M. Auslander, I. Reiten And S.O. Smal $\varnothing$. Representation Theory of Artin Algebras. Cambridge Studies in Advanced Mathematics № 36, Cambridge University Press, Cambridge (1995). MR 96c:16015

[3] E. Enochs. Injective and Flat Covers, Envelopes and Resolvents. Israel J. Math. 39 (1981), 189-209. MR 83a:16031

[4] E. Enochs. Torsion Free Covering Modules II. Arch. Math. (Basel) 22 (1971), 37-52. MR 44:1689

[5] E. Enochs, J.R. García Rozas and L. Oyonarte. Covering Morphisms. Submitted

[6] Jinzhong Xu. Flat Covers of Modules. Lecture Notes in Mathematics № 1634, Springer (1996).

Department of Mathematics, University of Kentucky, Lexington, Kentucky 405060027

E-mail address: enochs@ms.uky.edu

Departamento de Algebra y AnÁlisis Matemático, University of Almería, 04120 Almería, Spain

E-mail address: jrgrozas@ualm.es

Departamento de Algebra y Análisis Matemático, University of Almería, 04120 Almería, Spain

E-mail address: loyonart@ualm.es 